\title{
Au Catalyzing Control Release NO in vivo and Tumor Growth-Inhibiting Effect in Chemo- Photothermal Combination Therapy
}

This article was published in the following Dove Press journal: International Journal of Nanomedicine

\author{
Ying Zhang' \\ Tianfu Zhou' \\ Jian $\mathrm{Li}^{\prime}$ \\ Nuo Xu (iD) \\ Mingze Cai' \\ Hong Zhang ${ }^{2}$ \\ Qinfu Zhao ${ }^{3}$ \\ Siling Wang ${ }^{3}$ \\ 'Key Laboratory of TargetDrug Design \\ and Research, Ministry of Education, \\ Shenyang Pharmaceutical University, \\ Shenyang, I10016, People's Republic of \\ China; ${ }^{2}$ Van 'T Hoff Institute for \\ Molecular Sciences, University of \\ Amsterdam, Amsterdam, $1098 \mathrm{XH}$, the \\ Netherlands; ${ }^{3}$ Department of \\ Pharmaceutics, School of Pharmacy, \\ Shenyang Pharmaceutical University, \\ Shenyang, II0016, People's Republic of \\ China
}

Introduction: Aim to obtain a NO donor that can control released NO in vivo with the high

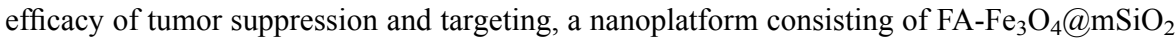
$-\mathrm{Au} / \mathrm{DOX}$ was constructed.

Methods: In vitro, the nanoplatform catalyzed NO's release with the maximum value of $4.91 \mu \mathrm{M}$ within $60 \mathrm{~min}$ at $43^{\circ} \mathrm{C} \mathrm{pH}=5.0$, which was increased by 1.14 times when the temperature was $37^{\circ} \mathrm{C}$. In vivo, $11.7 \mu \mathrm{g} \mathrm{Au}$ in the tumor tissue was found to catalyze S-nitrosoglutathione continuously, and $54 \mu \mathrm{M}$ NO was checked out in the urine.

Results and Discussion: The high concentration of NO was found to increase the apoptotic rate and to reduce tumor proliferation. In the chemo-photothermal combination therapy, the tumor inhibition rate was increased up to $94.3 \%$, and Au's contribution from catalyzing NO release NO was $8.17 \%$.

Keywords: thermotherapy, chemotherapy, NO control release, magnetic mesoporous silica nanocomposites

\section{Introduction}

The multifunctional therapeutic system with targeted drug delivery, imaging, and a combination therapy mode, which can greatly increase therapeutic efficacy and minimize the damage to normal tissues, has been extensively studied..$^{1-3}$ The integration of the thermo-chemo therapeutic system consisting of gold nanocomposites, ${ }^{4}$ carbon nanotubes, ${ }^{5}$ and magnetic mesoporous microspheres ${ }^{6}$ is an effective therapeutic system in vitro and vivo. Despite achievements that have been made, the integrated therapeutic systems usually loading an exogenous drug (doxorubicin, paclitaxel, and macromolecular protein), which commonly bring undesirable side effects, ineffective delivery to the tumor sites, and tumor cell resistance, ${ }^{7-9}$ are still facing difficult problems. Nitric oxide (NO) as a messenger molecule in vivo plays an important role in many physiological activities, such as cardiovascular, cerebrovascular, respiratory, nervous, and immune response. ${ }^{10} \mathrm{NO}$ presenting in low concentration exerts physiological function; however, $\mathrm{NO}$ with high concentration forms highly reactive substances $\left(\mathrm{N}_{2} \mathrm{O}_{3}, \mathrm{ONOO}^{-}\right.$, etc.), causing nitrification and oxidative stress, excessive consumption of glutathione (GSH) in cells, obstruction of mitochondria normal function, damage DNA and induce apoptosis. ${ }^{11-13}$ Given the dramatically opposite roles of NO causing by concentration, it is a good strategy to design a controlled release NO donor integrated into a multifunctional therapeutic system.
Correspondence: Qinfu Zhao; Siling Wang

Tel/Fax +86 2443520555

Email qinfuzhao@syphu.edu.cn;

silingwang@syphu.edu.cn
International Journal of Nanomedicine 2021:16 250I-2513

DovePress if in $>$ 
Several kinds of NO donors have been reported as drugs to investigate the anti-tumor efficacy. ${ }^{14-17}$ Unfortunately, those donors lack cancer cells targeting design for NO release in a controlled manner resulting in unsatisfactory tumor suppression and limiting their real application in treatment. Recently, some specific catalysts (such as $\mathrm{Cu}^{2+}, \mathrm{Fe}^{3+}, \mathrm{Au}$, etc.) have been proved for the NO generation whenever they come into contact with fresh blood serum; for example, S-nitroso serum albumin and S-nitrosoglutathione (GSNO) wide-spreading in blood will break S-NO bond to produce NO under the action of catalysts. $^{18,19}$ Among these catalysts, Au nanoparticles, which have near-infrared light absorption characteristics and can convert the absorbed light energy into heat energy, and meanwhile, activate heat-related pathways in tumor cells and induce expression of apoptotic proteins, ${ }^{20-23}$ draw our research interest. Also, it has been reported that magnetic gold nanocomposites could precisely accumulate in the tumor after a long period of blood circulation and significantly improve anti-tumor drugs' efficacy (doxorubicin, paclitaxel, and macromolecular protein) in the combination of thermo-chemotherapy. ${ }^{24-26}$ To design a multifunctional therapeutic system consisting of $\mathrm{Au}$ is an ideal solution for integrating controlled NO-release donors into a thermo-chemotherapy system.

To demonstrate $\mathrm{Au}$ can act as a NO donor catalyst in vivo chemotherapy by releasing NO in a thermally controlled manner and obtain satisfactory tumor suppression, the nano platform FA-MMSN-Au/DOX combining thermochemotherapy and multiple recognition mechanisms for tumor cells was constructed. Firstly, the aminated magnetic mesoporous silicon nanoparticles $\left(\mathrm{MMSN}_{\mathrm{N}} \mathrm{N}_{2}\right)$ were prepared by a thermal decomposition method and sol-gel method and then assembled with Au nanoparticles by electrostatic adsorption (MMSN-Au), and finally loaded with DOX and coupled with FA molecules (as shown in Figure 1A). Secondly, in vitro, the relationship between NO release amount catalyzed by MMSN-Au particles and temperature and GSNO concentration was measured. Thirdly, in vivo with a magnetic target and the near-infrared light radiation, the FA-MMSN-Au/DOX carrier accumulated at the tumor site and produced heat increasing tissue temperature; the NO released amount checked out in the urine. A contrastive combination therapy scheme was designed to investigate the effect of NO catalyzed by $\mathrm{Au}$ on tumor suppression. Under the same hyperthermia conditions, the effects of FAMMSN-Au/DOX and the FA-MMSN/DOX carrier on tumor growth at different stages were compared, and Au's contribution as NO donor catalyst was clarified. This provides a useful complement to Au's thermotherapy mechanism, and the results have never been reported before. Our work also provides a new strategy for integrating nitric oxide chemical biology and multi-modal combination therapy, such as Au thermo-chemotherapy.

\section{Materials and Methods Materials}

$\mathrm{FeCl}_{3} \cdot 6 \mathrm{H}_{2} \mathrm{O}$, sodium oleate, oleic acid were purchased from Tianjin Yongda Chemical Reagent Co., Ltd.; oleylamine (80-90\%) was purchased from Shanghai McLean Biochemical Technology Co., Ltd. Cetyl trimethyl ammonium bromide (CTAB) and ammonium nitrate were purchased from Beijing Chemical Reagent Co., Ltd.; n-hexane, absolute ethyl alcohol and sodium hydroxide (95\%) were purchased from Tianjin Damao Chemical Reagent factory; octadecene, N-(2-aminoethyl)-3-amopropyl trimethoxysilane (EDS), tetraethoxysilane (TEOS) were purchased from Beijing Balinwei Reagent Co., Ltd.; Polyoxyethylene-polypropylene-polyoxyethylene copolymer (F127) was purchased from Germany BASF Company; chloroauric acid $\left(\mathrm{HAuCl}_{4} \cdot 3 \mathrm{H}_{2} \mathrm{O}, 99.99 \%\right)$ was purchased from Alfa Aesha (China) Chemical Co., Ltd.; folic acid (FA, $\geq 97 \%$ ), doxorubicin hydrochloride (DOX, $\geq 98 \%$ ) and S-Nitrosylglutathione (GSNO) was obtained from Aladdin Bio-chem Technology Co., Ltd.; Griess reagent Shanghai Biyuntian Biotechnology Co., Ltd. 1-hydroxybenzotriazole (HOBT) and O-benzotriazole tetramethyl urea hexafluorophosphate (HBTU) were purchased from Gill Biochemical (Shanghai) Co., Ltd. All chemicals were analytical reagent grade and used without further purification.

\section{Construction of the Multifunctional Platform FA-MMSN-Au}

$\mathrm{Fe}_{3} \mathrm{O}_{4}$ magnetite nanoparticles (MNP) were synthesized using the thermal decomposition method established by Park et al. ${ }^{27} \mathrm{Fe}_{3} \mathrm{O}_{4} @ \mathrm{mSiO}_{2}-\mathrm{NH}_{2}$ magnetic mesoporous silica nanocomposites $\left(\mathrm{MMSN}-\mathrm{NH}_{2}\right)$ were prepared according to the method reported by our group. ${ }^{28} \mathrm{Au}$ nanoparticles were prepared according to the literature. ${ }^{29}$ The above MMSN-NH $\mathrm{N}_{2}$ absolute ethyl alcohol solution ( $1 \mathrm{~mL}, 20 \mathrm{mg} / \mathrm{mL}$ ) was added to the Au solution $(10 \mathrm{~mL}, 2.4 \mathrm{mg} / \mathrm{mL})$ and kept stirring for $1 \mathrm{~h}$. After centrifugation (4500 rpm/min, $10 \mathrm{~min}$ ), the magnetic gold nanocomposites (MMSN-Au) were collected and 


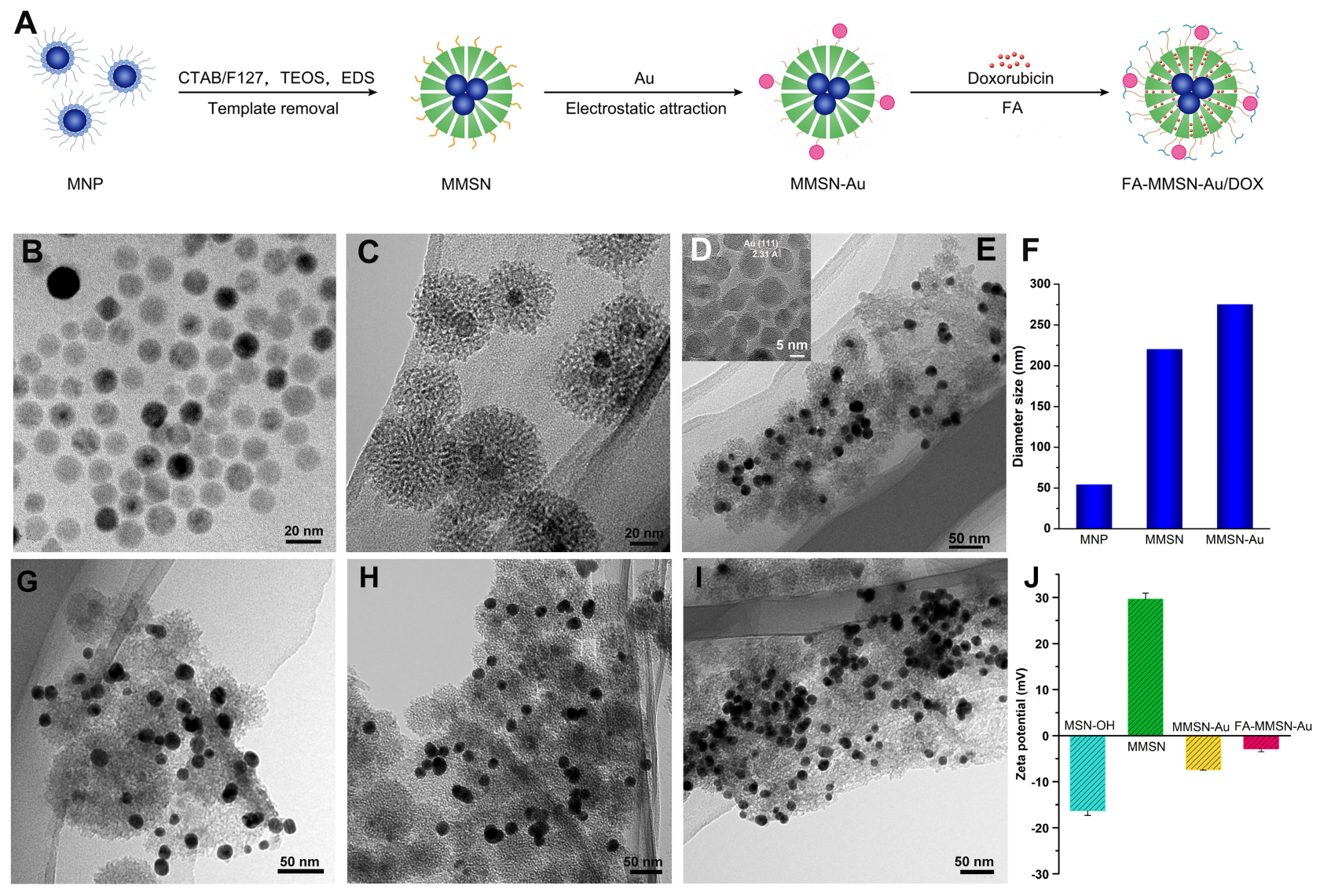

Figure I (A) Scheme of the multifunctional platform; TEM images of (B) MNP, (C) MMSN, (D) HRTEM image of Au nanoparticles, (E) MMSN-Au nanocomposites; MMSNAu with different dose of EDS (G) $20 \mu \mathrm{L},(\mathbf{H}) 40 \mu \mathrm{L}$, (I) $80 \mu \mathrm{L}$; (F) the size distribution of MNP, MMSN, and MMSN-Au. The diameter of MNP comes from reference, ${ }^{32}(\mathbf{J})$ Zeta potential change.

rinsed with ethanol two times. FA $(10 \mathrm{mg})$ was dissolved in dimethyl sulfoxide (DMSO, $5 \mathrm{~mL}$ ). Then, 1-ethyl(3-dimethyl aminopropyl) carbodiimide hydrochloride (EDC, $200 \mathrm{mg}$ ) and n-hydroxysuccinimide (NHS, $160 \mathrm{mg}$ ) were added for excitation $3 \mathrm{~h}$. MMSN-Au (100 mg) nanoparticles dispersing in DMSO $(20 \mathrm{~mL})$ were added to the above solution and reacted $24 \mathrm{~h}$ at room temperature. The final carrier of FA-MMSN-Au was obtained by centrifugation.

\section{Thermal Properties Study}

The test solutions were prepared by dispersing accurate amounts of the FA-MMSN-Au composites in PBS $(\mathrm{pH}=7.4)$ solutions. The prepared solutions $(1 \mathrm{~mL})$ with different concentrations were introduced into cuvettes and exposed to the NIR light laser $\left(808 \mathrm{~nm}, 0.27 \mathrm{~W} / \mathrm{cm}^{2}\right)$. For another group, series concentration FA-MMSN-Au solutions $(1 \mathrm{~mL})$ were placed in a high-frequency induction heating instrument with different alternating current. The temperature of the solutions was recorded using a thermal imager.

\section{Drug Loading and in vitro Release}

First, DOX (4 mg) and MMSN-Au (20 mg) composites were added to PBS $(2.0 \mathrm{~mL}, \mathrm{pH}=7.4)$ solution, followed by mixing and shaking dispersion for $24 \mathrm{~h}$. Second, the composites were washed with $\mathrm{pH}$ 7.4 PBS solution to remove the DOX adsorbing on the support surface until colorless. The composites were then collected and recorded as FA-MMSN-Au/DOX; the supernatant was also collected and measured at a wavelength of $480 \mathrm{~nm}$. According to the literature method, ${ }^{30}$ the load capacity was calculated as follows: $\mathrm{EE}=\frac{M_{\text {loadingDOX }}}{M_{\text {TotalDOX }}} \times 100 \%$

The drug release behavior was investigated as follows: $4 \mathrm{mg}$ MMSN-Au/DOX was dispersed in $10 \mathrm{~mL} \mathrm{pH} 5.0$ and 7.4 PBS in two different dispersion media (simulating tumor tissue and normal tissue environment, respectively), and placed in a $37^{\circ} \mathrm{C}$ air bath shaker at $120 \mathrm{rpm}$ shaking. At a specific time $(0.5,1,2,3,4$, and $6 \mathrm{~h})$, the release 
medium's absorbance was measured at $480 \mathrm{~nm}$. The drug release behavior of MMSN-Au/DOX was measure in the same procedure.

GSNO PBS solution was used to simulate plasma, and gold nanoparticles in MMSN-Au composites could catalyze GSNO release NO, converted to nitrite or nitrate in PBS solution. The Griess kit was used to detect MMSN-Au carrier performance catalyzing S-nitrosoglutathione (GSNO) to release of NO. ${ }^{31}$ According to the instruction of the Griess kit, the NO standard curve was determined. Next, $2 \mathrm{~mL}$ PBS solution containing $300 \mu \mathrm{L}$ GSNO $(1.2 \mathrm{mM})$ and $1 \mathrm{~mL}$ of MMSN-Au nanocomposite aqueous solution $(12 \mathrm{mg} / \mathrm{mL})$ was mixed in the sealed dialysis bag immersing in a $35 \mathrm{~mL}$ PBS solution at $37^{\circ} \mathrm{C}$. After dialysis for a different time, $1 \mathrm{~mL}$ of PBS solution outside the dialysis bag was taken out for NO test with maintaining the constant volume of PBS solution. The experiments under the condition of $2.4 \mathrm{mM}$ and $4.8 \mathrm{mM} \mathrm{GSNO}$, and $25^{\circ} \mathrm{C}$ and $43^{\circ} \mathrm{C}$ were also measured with the above procedure.

\section{Cell Culture and Cellular Uptake}

MCF-7 cells were provided from Shanghai Cell Institute (Shanghai, China). MCF-7 cells maintained in RPMI 1640 supplemented with FBS (10\%), penicillin (1\%), and streptomycin $(1 \%)$ in $5 \% \mathrm{CO}_{2}$ at $37^{\circ} \mathrm{C}$. The medium was routinely changed every 2 days, and the cells were separated by trypsinization before reaching confluence. For microscopic analysis, MCF-7 cells were seeded in round glass coverslips at $5 \times 10^{4}$ cells/well in 24-well plates. Then, DOX solution and FA-MMSN-Au/DOX (equal to 5 $\mu \mathrm{g} / \mathrm{mL}$ DOX) were added into the corresponding wells. After $2 \mathrm{~h}$, the cells were washed with cold PBS, fixed with formaldehyde, and stained with DAPI. The fixed cells were observed by confocal laser scanning microscopy (CLSM). A quantitative evaluation of the cellular uptake was performed on the flow cytometry technique (FCM). MCF-7 cells were seeded in 6-well plates and incubated with DOX solution and FA-MMSN-Au/DOX (equal to 5 $\mu \mathrm{g} / \mathrm{mL}$ DOX). Subsequently, the cells were rinsed, trypsinized and resuspended in $400 \mu \mathrm{L}$ PBS. The mean fluorescence intensity (MFI) was measured using FCM (FACSCalibur, Becton Dickinson, USA). In the competition experiment, the MCF-7 cells were incubated with $2 \mathrm{mg} / \mathrm{mL}$ FA for $2 \mathrm{~h}$ before the nanoparticles were added.

\section{Therapy Program}

Experiments involving animals were carried out under the following information. The ethics approval number was
SYPU-IACUC-C2017, the authorizing institution name was Animal Ethics Committee of Shenyang Pharmaceutical University, and the use of animals in research was followed to NC3Rs ARRIVE guidelines.

Mouse sarcoma S180 cells were provided from Shanghai Cell Institute (Shanghai, China) and were cultured in RPMI 1640 medium containing $10 \%$ FBS in $5 \% \mathrm{CO}_{2}$ at $37^{\circ} \mathrm{C}$. Kunming mice were subcutaneous inoculation of $\mathrm{S} 180$ cells $\left(1 \times 10^{7}\right.$ cells $)$ into the right flanks. S180 tumorbearing mice were randomly divided into several groups consisting of six mice each. Blank control group: no treatments were given. DOX control group: $0.2 \mathrm{mg} / \mathrm{mL}$ DOX $0.2 \mathrm{~mL}$ was injected into the tail vein. FA-MMSN-Au/DOX magnetic targeting chemotherapy experimental group (T):

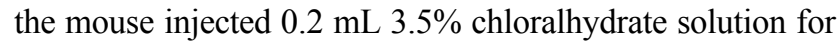
abdominal anesthesia and was fixed on the thermotherapy plate. After tail vein inception of $0.2 \mathrm{~mL} 12 \mathrm{mg} / \mathrm{mL}$ FAMMSN-Au/DOX carrier, 0.3T permanent magnet was used for the 30 min's target. FA-MMSN-Au/DOX magnetic targeting chemo-magneto therapy experimental group $(\mathrm{T}+\mathrm{MT})$ : the same treatment as FA-MMSN-Au/DOX (T) experimental group was used. The mouse was then put in the magnetic induction coil; the tumor site was located at the center of the magnetic induction coil with alternating current $25 \mathrm{~A}$ to maintain $43^{\circ} \mathrm{C}$ for $30 \mathrm{~min}$. FA-MMSN/DOX magnetic targeted chemo-magneto thermal experimental group $(\mathrm{T}+\mathrm{MT})$ : the same treatment as FA-MMSN-Au/DOX (T+MT). FAMMSN-Au/DOX magnetic targeting chemo-photo thermal experiment group ( $\mathrm{T}+\mathrm{PT})$ : After anesthesia, tail vein injection, and magnetic target, the mouse was put under the 808 $\mathrm{nm}$ near-infrared laser emitter with a power density of 0.27 $\mathrm{W} / \mathrm{cm}^{2}$, and the irradiation of the tumor site was heated to $43^{\circ}$ $\mathrm{C}$ for $30 \mathrm{~min}$. The thermal imager recorded the temperature.

\section{In vivo HE Staining Observation}

HE staining microscopic observation of heart, lung, liver, spleen, and kidney specimens of mice after $24 \mathrm{~h}$ of injection with FA-MMSN-Au/DOX $(12 \mathrm{mg} / \mathrm{mL}, 0.2 \mathrm{~mL})$ was used as a control group to observe histopathology.

\section{Detection of NO Content in Urine}

The measurement was performed using a Griess kit. Blank control group: no treatment was given; $0.5 \mathrm{~mL}$ urine was taken daily for measurement. FA-MMSN-Au/DOX magnetic targeted chemo-photo thermal experimental group ( $\mathrm{T}$ $+\mathrm{PT})$ : After each treatment, it was measured for $0.5 \mathrm{~mL}$ of urine within 4-24 h. 


\section{Results}

\section{Materials Synthesis and Characterization}

The integrated therapeutic system was synthesized as the scheme in Figure 1A and the characterization is described in the Supplementary materials. It can be seen that the MNP magnetic nanoparticles were uniform solid spheres with a particle size of about $14 \mathrm{~nm}$ and good dispersibility (Figure 1B). The MMSN carrier was spherical with a uniform size of $50 \mathrm{~nm}$, and the thickness of the mesoporous silicon shell was about $15-20 \mathrm{~nm}$. The magnetic MNP nanoparticles were embedded in the center of the silica shell. To get better magnetic and thermal properties, we increased the mass ratio of MNP and TEOS in synthesizing magnetic mesoporous silica nanocomposites, which resulted in multicores of MNP embedding in the center of the silica shell (Figure 1C). The MMSN-Au nanocomposites showed a certain degree of aggregation, and a small amount of gold nanoparticles were adsorbed on the surface of MMSN (Figure 1E). The gold nanoparticles with a particle size of about $8 \mathrm{~nm}$ (Figure 1D) can be adsorbed on the surface of MMSN in a controlled manner. The silane coupling agent $\mathrm{N}$-(2-aminoethyl)-3-aminopropyl trimethoxysilane (EDS) was used as the adjusted reagent to modify the Zeta potential of MMSN (Figure S1). As shown in Figure $1 \mathrm{G}$ and H, when the amount of EDS was $20 \mu \mathrm{L}$ and $40 \mu \mathrm{L}$, the number of gold nanoparticles adsorbed by MMSN was similar; when the amount of EDS was $80 \mu \mathrm{L}$, the MMSN-Au carrier's dispersibility decreased, and the amount of Au absorbed by MMSN increased significantly (Figure 1I). The size distribution of MNP, MMSN, and MMSN-Au was evaluated by DSL (Figure 1F). The change of particle size during the preparation process was consistent with the composite structure observed by TEM. The synthesis procedure was also proved by the Zeta potential (Figure 1J). MSN with hydroxyl showed $-16.2 \mathrm{mV}$ and MMSN modifying with amino groups showed $30.0 \mathrm{mV}$, which changed little with the amount of EDS; after coupling with Au stabilized by citric acid, the potential of MMSN decreased to $-7.6 \mathrm{mV}$. MMSN-Au loading FA with amino groups accordingly resulted in the potential increase to $-2.877 \mathrm{mV}$.

Figure 2A shows the XRD patterns of MNP, MMSN, and MMSN-Au. The MNP diffraction pattern was consistent with the standard $\mathrm{Fe}_{3} \mathrm{O}_{4}$ PDF card (19-0629). The characteristic diffraction peaks of $\mathrm{Fe}_{3} \mathrm{O}_{4}$ nanoparticles appeared at $2 \theta=35.40,42.92,56.97$, and $62.22^{\circ}$, which proved that $\mathrm{Fe}_{3} \mathrm{O}_{4}$ nanoparticles were formed. As for the MMSN diffraction pattern, the characteristic peaks of $\mathrm{Fe}_{3} \mathrm{O}_{4}$ still maintain, which indicates that the crystal structure of $\mathrm{Fe}_{3} \mathrm{O}_{4}$ nanoparticles does not change during the $\mathrm{SiO}_{2}$ coating. In the MMSN-Au diffraction pattern, Au nanoparticles' diffraction peaks appeared at
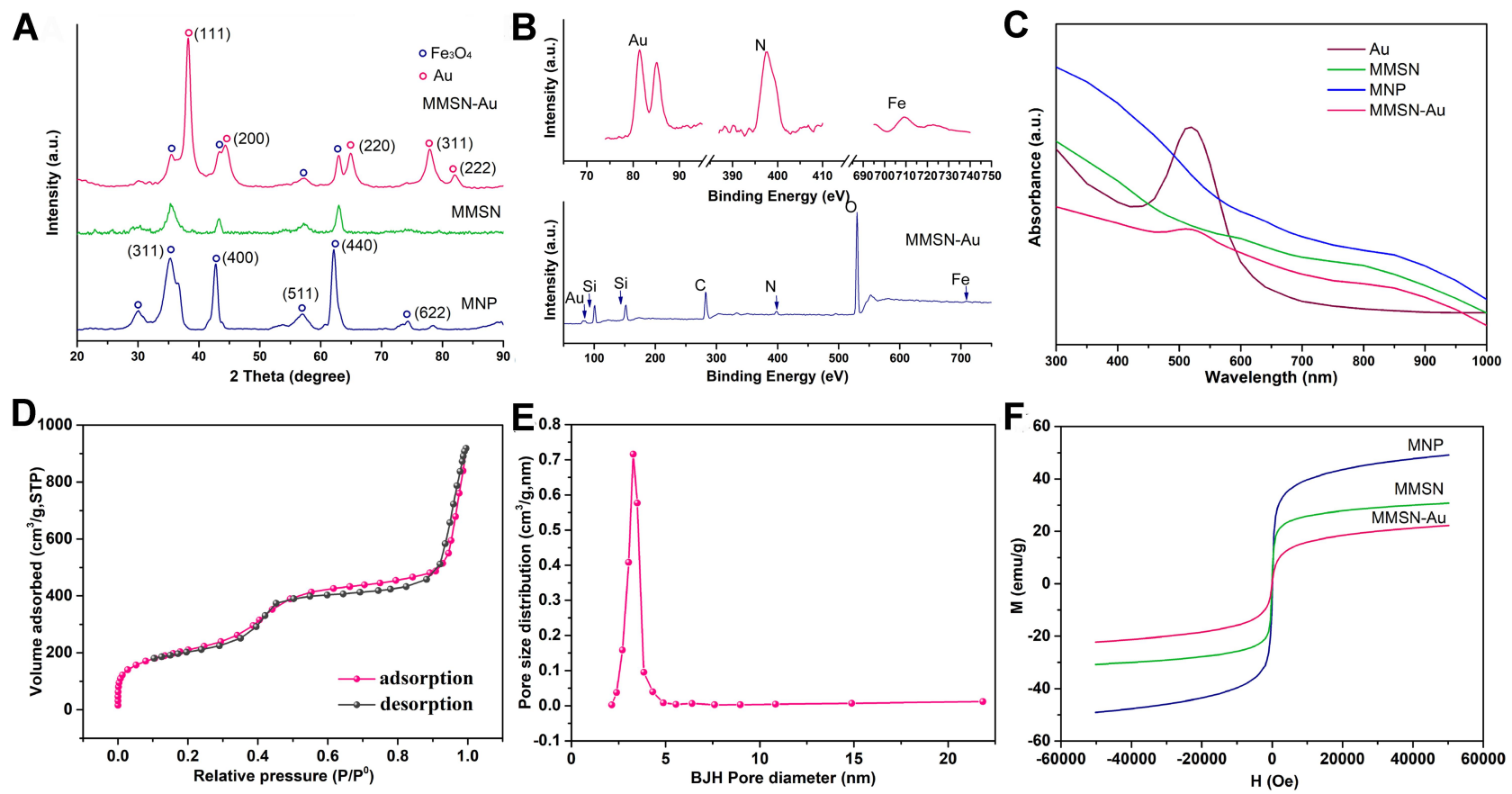

Figure 2 (A) XRD patterns of MNP, MMSN, and MMSN-Au; (B) XPS spectrum of MMSN-Au; (C) UV-vis spectrum of Au, MNP, MMSN, and MMSN-Au; (D) N 2 adsorptiondesorption isotherm; (E) pore size distribution curve of MMSN; (F) hysteresis loop of MNP, MMSN and MMSN-Au nanocomposites, T=298 K. 
$2 \theta=38.17,44.24,64.90,77.70$, and $81.82^{\circ}$, indicating that $\mathrm{Au}$ (17-0629) was successfully prepared, also, the crystal structure of $\mathrm{Fe}_{3} \mathrm{O}_{4}$ nanoparticles had not changed during $\mathrm{Au}$ adsorption. The binding energies and atomic percentage of MMSN-Au were analyzed by XPS (Figure 2B and Table 1). The two peaks of 709.2 and $722.3 \mathrm{eV}$ in the carrier coincided with $\mathrm{Fe} 2 \mathrm{p} 1 / 2$ and $\mathrm{Fe} 2 \mathrm{p} 3 / 2$, two different state standard peaks. The peak of the binding energy of $397.7 \mathrm{eV}$ indicated the binding energy spectrum of $\mathrm{N}$. The peaks in 81.4 and $85.1 \mathrm{eV}$ were Au's characteristic peaks. The atom ratio $\mathrm{Si}$ : $\mathrm{C}$ was reduced slightly after the grafting process, indicating that $\mathrm{Au}$ stabilized by citric acid coupled with MMSN. In summary, the magnetic gold nanocomposites were successfully constructed. The UV absorbance spectrum is shown in Figure 2C. The absorbance peak of $480 \mathrm{~nm}$ was the characteristic of $\mathrm{Au}$ nanoparticles. After coupling with MMSN, Au's absorbance intensity declined but remained, indicating that the MMSN assembled with Au.

The adsorption-desorption isotherms and pore size distributions of MMSN were characterized by $\mathrm{N}_{2}$ adsorption-desorption analysis, as shown in Figure 2D and E. $\mathrm{N}_{2}$ adsorption-desorption isotherm showed the shape and characteristics of the type IV isotherm with a hysteresis loop that confirmed the typical mesoporous structure inside the carrier. The pore size distribution curve of MMSN showed a sharp peak distribution indicating that the pore structure of the MMSN carrier is uniform and regular. The $\mathrm{S}_{\mathrm{BET}}$, pore size distribution $\left(D_{p}\right)$, and total pore volume $\left(V_{t}\right)$ were shown in Table 2. The single-point BET specific surface area of the carrier was $734.956 \mathrm{~m}^{2} / \mathrm{g}$, the cumulative pore volume of BJH adsorption was $1.41 \mathrm{~cm}^{3} / \mathrm{g}$, and the calculated

Table I Elemental Analysis of MMSN and MMSN-Au (Atomic Percentage)

\begin{tabular}{|l|c|c|c|c|c|c|}
\hline Sample & $\mathbf{F e}(\%)$ & $\mathbf{S i}(\%)$ & $\mathbf{O}(\%)$ & $\mathbf{C}(\%)$ & $\mathbf{N}(\%)$ & $\mathbf{A u}(\%)$ \\
\hline MMSN & 0.35 & 24.45 & 45.62 & 27 & 2.59 & - \\
MMSN-Au & 0.31 & 22.55 & 41.66 & 32.28 & 2.89 & 0.31 \\
\hline
\end{tabular}

Table 2 Size Distribution and Mesopore Structural Parameters of MMSN

\begin{tabular}{|l|c|c|c|}
\hline Sample & $\mathbf{S}_{\text {BET }}\left(\mathbf{m}^{2} / \mathbf{g}\right)$ & $\mathbf{V}_{\mathbf{t}}\left(\mathrm{cm}^{3} / \mathbf{g}\right)$ & $\mathbf{W}_{\mathbf{B J H}}(\mathbf{n m})$ \\
\hline MMSN & 734.96 & 1.41 & 3.2 \\
\hline
\end{tabular}

pore size of BJH desorption data was $3.2 \mathrm{~nm}$. Figure $2 \mathrm{~F}$ shows the magnetic properties of MNP, MMSN, and MMSN-Au nanocomposites. All MNP, MMSN, and MMSN-Au nanocomposites exhibited paramagnetic characteristics at $\mathrm{T}=298 \mathrm{~K}$. The saturated magnetic strength of the MNP nanoparticles decreased from 49.1 to 30.8 and further to $22.3 \mathrm{emu} / \mathrm{g}$ after coating with $\mathrm{SiO}_{2}$ and $\mathrm{Au}$ nanoparticles.

\section{NO Catalysis Release and DOX Release}

Under the conditions of simulating the tumor's acidic environment ( $\mathrm{pH}$ 5.0 PBS) and plasma ( $\mathrm{pH}$ 7.4 PBS) in vitro, it can be seen from Figure $3 \mathrm{~A}-\mathrm{E}$ that the reaction of GSNO to release NO catalyzed by $\mathrm{Au}$ was positively correlated with GSNO concentration and temperature. In tumor tissue acid condition, as the GSNO concentration increased from $300 \mu \mathrm{L}$ to $1200 \mu \mathrm{L}$, the release amount of $\mathrm{NO}$ increased from $4.36 \mu \mathrm{M}$ to 5.85 $\mu \mathrm{M}$ in about $60 \mathrm{~min}$ (Figure $3 \mathrm{D}$ ); as the temperature increased from 37 to $43^{\circ} \mathrm{C}$, the $\mathrm{NO}$ release rate and amount gradually increased, reaching an equilibrium state $4.91 \mu \mathrm{M}$ in about $60 \mathrm{~min}$ (Figure 3E). At the hyperthermia temperature of $43^{\circ} \mathrm{C}$ for $30 \mathrm{~min}$, the total amount of NO produced by $\mathrm{Au}$ catalytic release was 1.14 times that of released at normal body temperature. Compared with the normal tissue condition, the NO release amount increased by $28.6 \%$ in the tumor, indicated in Figure 3C. Therefore, the carrier's performance to catalyze the release of NO from GSNO has a dual response to weak acid conditions and hyperthermia temperature. It can be inferred that in the tumor tissues treated with photothermal therapy, within $60 \mathrm{~min}$, the release of NO is significantly greater than that of normal tissues without thermotherapy.

The loaded and released DOX was calculated by the standard curve of DOX: A=0.0204C-0.0276. The drug encapsulating of MMSN-Au for $24 \mathrm{~h}$ was $21.0 \%$. The $-\mathrm{COOH}$ group of $\mathrm{DOX}$ and the $\mathrm{Si}-\mathrm{OH}$ group on the surface of the mesoporous pore wall may interact with each other in the form of hydrogen bonding, promoting the loading of DOX in the mesoporous channel. The in vitro DOX release behavior of MMSN-Au/DOX is shown in Figure 3F. The cumulative release amount of DOX from MMSN-Au/DOX in $\mathrm{pH} 5.0$ and 7.4 PBS solution was $27.8 \%$ and $19.9 \%$. The release amount of DOX from FA-MMSN-Au/DOX in pH 7.4 PBS solution was $6.5 \%$, which was significantly lower than that of MMSN$\mathrm{Au} / \mathrm{DOX}$ because the modification of FA molecules had a steric hindrance leading to the release of DOX slowed. 

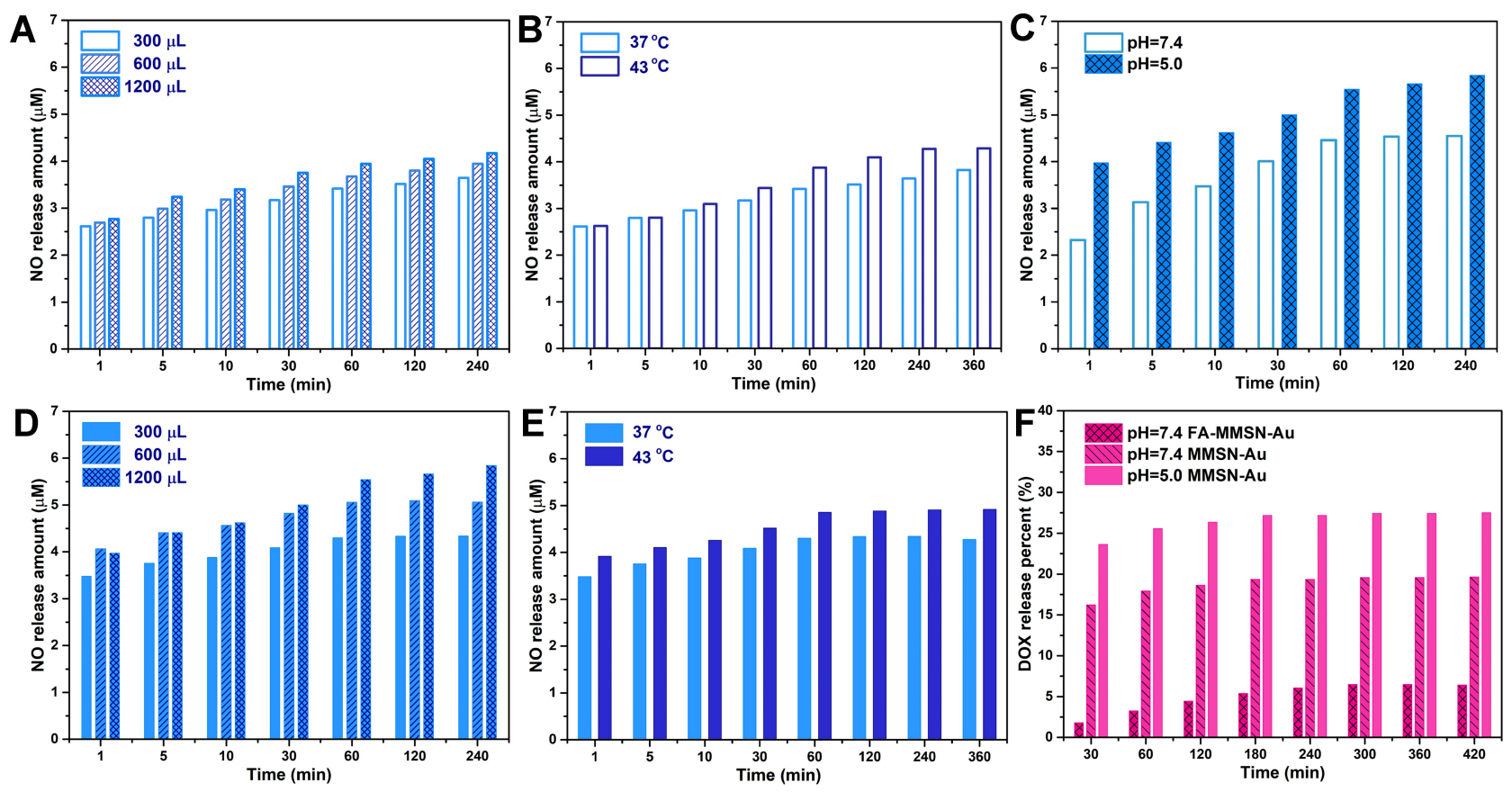

Figure $3 \mathrm{NO}$ release behaviors of MMSN-Au $(1.2 \mathrm{mg} / \mathrm{mL}, \mathrm{I} \mathrm{mL})(\mathbf{A})$ in different GSNO concentrations at $37^{\circ} \mathrm{C}$ and $\mathrm{pH}=7.4 \mathrm{PBS}$ solution; $(\mathbf{B})$ at different temperatures in $300 \mu \mathrm{L}$ GSNO and $\mathrm{pH}=7.4$ PBS solution; (C) DOX release behaviors in $37^{\circ} \mathrm{C}, \mathrm{pH}=7.4$ and $5.0 \mathrm{PBS}$ solution; (D) $37^{\circ} \mathrm{C}$ and $\mathrm{pH}=5.0 \mathrm{PBS}$ solution; (E) $300 \mu \mathrm{L}$ GSNO and $\mathrm{pH}=5.0$ PBS solution; (F) DOX release behaviors.
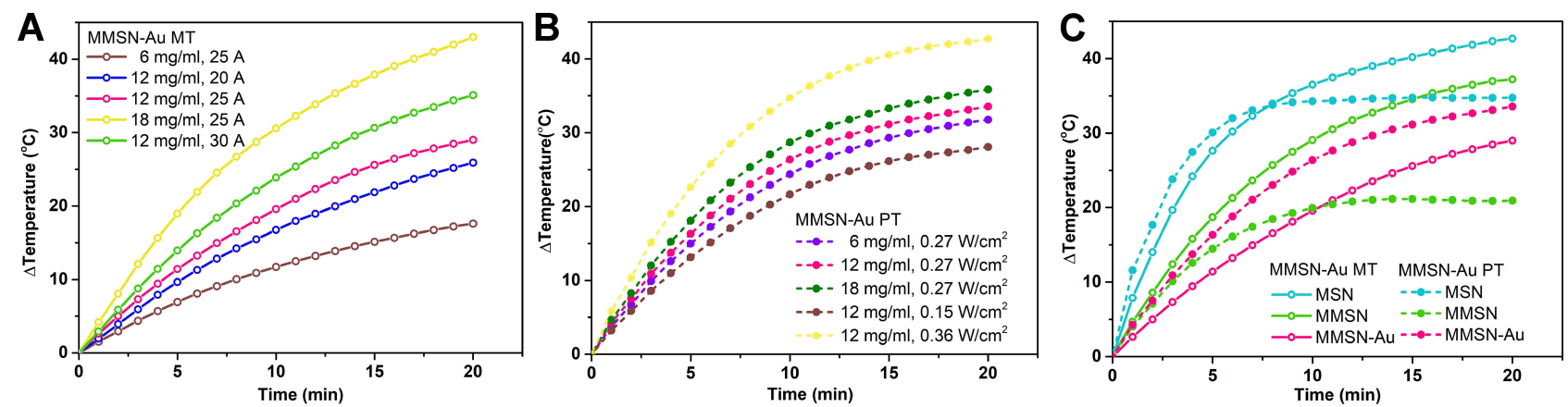

Figure 4 (A) Magnetothermal heating curves of MMSN-Au nanocomposites, (B) photothermal heating curves of MMSN-Au nanocomposites under the 808 nm laser radiation, (C) the heating curves of MNP, MMSN and MMSN-Au with the same concentration of $12 \mathrm{mg} / \mathrm{mL}, \mathrm{l}=25 \mathrm{~A}, \mathrm{~d}=0.27 \mathrm{~W} / \mathrm{cm}{ }^{2}, \mathrm{~V}=1 \mathrm{~mL}$.

\section{Magnetothermal and Photothermal Properties}

It can be seen from Figure 4A that the medium containing a higher amount of MMSN-Au generated a more significant temperature increase. For example, the sample of $12 \mathrm{mg} / \mathrm{mL}$ was heated up $29.1^{\circ} \mathrm{C}$ in $20 \mathrm{~min}$ at the current 25 A (Figure 4A). When the current increased from 20 A to $30 \mathrm{~A}$, the sample solution's temperature $(12 \mathrm{mg} / \mathrm{mL})$ changed from $17.7^{\circ} \mathrm{C}$ to $43.1^{\circ} \mathrm{C}$ (Figure $4 \mathrm{~A}$ ). Basing on the withstanding current values and MMSN-Au hemolysis rate for the mice, the condition of current $25 \mathrm{~A}$ and concentration $12 \mathrm{mg} / \mathrm{mL}$ were selected in the following magnetothermal treatment. It can be seen from Figure 5B that the medium containing a higher amount of MMSN-Au generated a more significant temperature increase. The $12 \mathrm{mg} / \mathrm{mL}$ sample was heated up at $33.6^{\circ} \mathrm{C}$ in $20 \mathrm{~min}$ at the density of $0.27 \mathrm{~W} / \mathrm{cm}^{2}$. When the optical density increased from 0.15 to $0.36 \mathrm{~W} / \mathrm{cm}^{2}$, the sample solution's temperature $(12 \mathrm{mg} / \mathrm{mL})$ varied from $28.0^{\circ} \mathrm{C}$ to $42.7^{\circ} \mathrm{C}$ (Figure 4B). In general, MMSN-Au nanocomposites exhibited obvious photothermal performance and magnetothermal behavior.

Aim to understand the difference between the magneto and photothermal behavior and its effect factors, the 


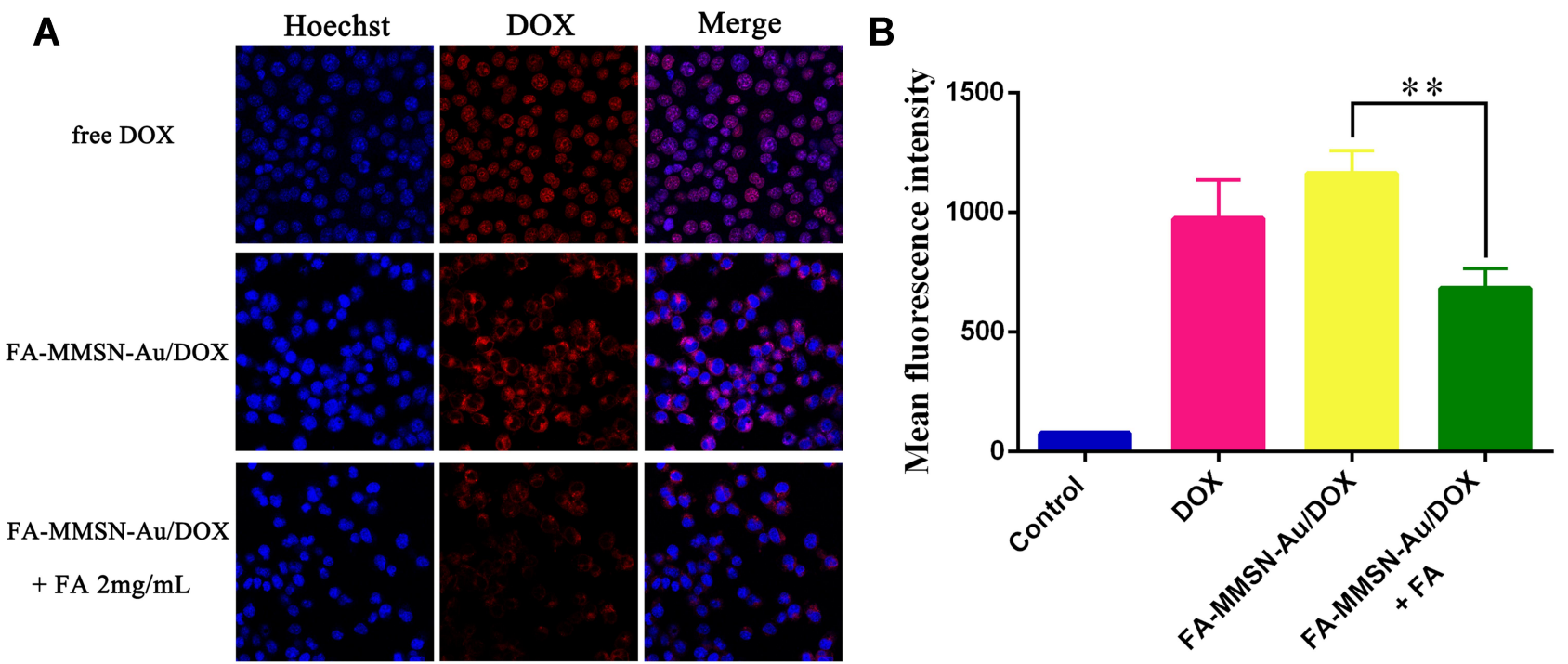

Figure 5 (A) CLSM images and (B) FCM analysis of MCF-7 cells incubated with free DOX, and FA-MMSN-Au/DOX and preincubated with FA for $2 \mathrm{~h}$ before the addition of FA-MMSN-Au/DOX

Note: $* * P<0.05$

changes in magneto/photothermal properties of MNP and MMSN were also measured and further compared with the behavior of MMSN-Au carriers. As shown in Figure 4C, the MNP itself had excellent photothermal absorption properties that could be rapidly heated up at $34^{\circ} \mathrm{C}$ within $8 \mathrm{~min}$. After coating with the $\mathrm{SiO}_{2}$ shell layer, which had no absorption of light, the heating rate of the carrier MMSN decreased (Figure 4C). When Au is coupled with MMSN, the photothermal effect increased to a certain extent, which may be attributed to Au's surface electron oscillation after

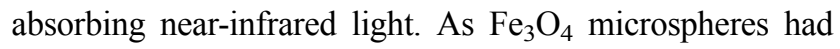
stronger absorption in the near-infrared region than $\mathrm{Au}$, and MNP had better photothermal conversion under nearinfrared light, ${ }^{32,33} \mathrm{MNP}$ temperature heating rate was higher than MMSN-Au under PT. However, things were different for magnetothermal properties because $\mathrm{Au}$ nanoparticles could not generate heat in an alternating electric field. The coupling of Au led to reducing the magnetothermal properties of the carrier. Therefore, for the composite carrier MMSN-Au, the photothermal performance was superior to the magnetocaloric property under the experiment condition. The light-to-heat conversion efficiency of MMSN-Au was $24.04 \%$ that was calculated from Figure S2.

\section{Cellular Uptake}

FA receptors over-expressed MCF-7 cells were used to evaluate the cellular uptake of FA-MMSN-Au/DOX nanoparticles. ${ }^{34,35}$ As shown in Figure 5A, free Dox was mainly localized in the nuclei due to its high affinity with
DNA. The red fluorescence of DOX appeared in the cytoplasm for FA-MMSN-Au/DOX group, and the fluorescence intensity markedly decreased after preincubation with FA for $2 \mathrm{~h}$, indicating that the target-specific endocytosis of FA-MMSN-Au/DOX was mediated by FA receptors. Furthermore, FCM was used to quantificationally measure the cellular uptake of FA-MMSN-Au/DOX (Figure 5B). The MFI was dramatically reduced compared with the untreated FA-MMSN-Au/DOX group with a $\mathrm{p}$ value of 0.003 , when MCF-7 cells were pretreated with $2 \mathrm{mg} / \mathrm{mL}$ FA for $2 \mathrm{~h}$. These results indicated that the modified FA on the surface of MMSN-Au could improve the cellular uptake in FA receptors over-expressed MCF-7 cells.

\section{Materials Cytotoxicity}

Before the hyperthermia treatment, the histocompatibility of the FA-MMSN-Au/DOX carrier was investigated by HE staining. As shown in Figure 6, the liver structure of the FAMMSN-Au/DOX group (Figure 6B) was slightly different from the saline control group (Figure 6B1), the accumulation of FA-MMSN-Au/DOX was observed in the liver cells. The hepatocytes were polygonally arranged in a stripe shape around the central vein, and the hepatocytes were free from degeneration and necrosis. The FA-MMSN-Au/DOX did not show obvious pathology reactions of the heart by comparing Figure $6 \mathrm{~A}$ and $\mathrm{A}_{1}$, spleen (Figure $6 \mathrm{C}$ and $\mathrm{C}_{1}$ ), lung (Figure 6D and $\mathrm{D}_{1}$ ), and kidney (Figure $6 \mathrm{E}$ and $\mathrm{E}_{1}$ ). Therefore, the carrier was histocompatibility. The constructed FA-MMSN-Au/DOX 


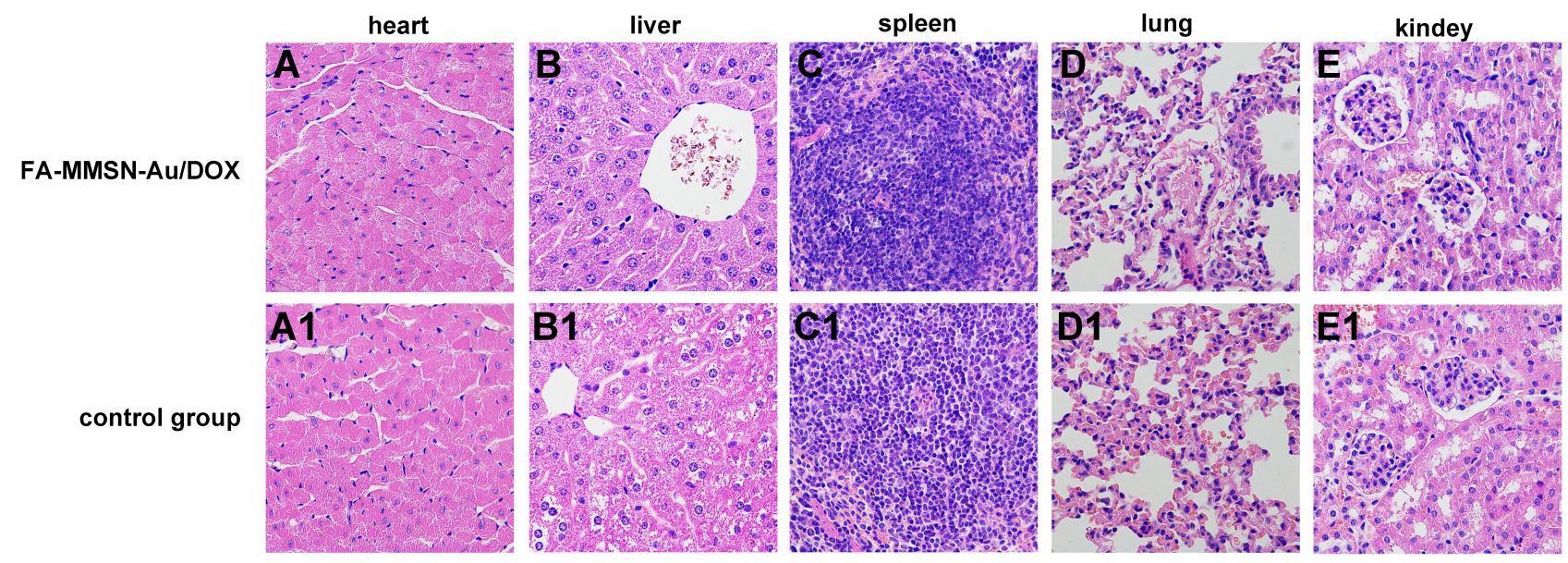

Figure 6 Cell HE staining results of experimental group with tail vein injection $0.2 \mathrm{~mL} 12 \mathrm{mg} / \mathrm{mL}$ FA-MMSN-Au/DOX after $24 \mathrm{~h}$ (A-E) and the control group (Al-EI). (A) Heart; (B) liver; (C) spleen; (D) lung; and (E) kidney in experimental group and the control group.

carrier was safe for blood; the adsorption capacity of FAMMSN-Au/DOX to BSA was $12.25 \%$, which can avoid the interference of protein to a certain extent; the systemic acute toxicity of the FA-MMSN-Au/DOX was negative (Figure S3, $\underline{\text { Table S1 }}$ and Table S2).

\section{In vivo Magneto/Phototherapy and NO in situ Chemotherapy Evaluation}

Multi therapy was used on tumor-bearing mice, as shown in Figure 7A. The tumor volume of experiment mice was recorded to evaluate the NO in situ chemotherapy effect and the combination magneto/photothermal therapeutic effect. The inhibition rate was calculated from the relative change of tumor volume. The tumor volume changes with time in each experimental group are shown in Figure 7B. Compared with the tumor growth of the saline group, the tumor growth was inhibited in the DOX group,
FA-MMSN-Au/DOX (T) group, FA-MMSN/DOX (T $+\mathrm{MT})$ group, FA-MMSN-Au/DOX (T+MT), and FAMMSN-Au/DOX $(\mathrm{T}+\mathrm{PT})$ group. As shown in Figure $7 \mathrm{C}$, compared with the tumor inhibition rates of DOX chemotherapy group 43.4\%, FA-MMSN-Au/DOX (T) group, FA-MMSN-Au/DOX (T+MT) group, FA-MMSN /DOX (T+MT), and FA-MMSN-Au/DOX (T+PT) group exhibited higher tumor inhibition rate with the value of $69.0 \%, 79.7 \%, 71.6 \%$, and $94.3 \%$, respectively. The tumor inhibition rate of FA-MMSN-Au/DOX (T+MT) was $10.7 \%$ higher than that of FA-MMSN-Au/DOX (T), which can be ascribed to the contribution of magnetic hyperthermia to tumor suppression. The tumor inhibition rate of FA-MMSN-Au/DOX (T+PT) was $25.3 \%$ higher than that of FA-MMSN-Au/DOX (T) and 14.6\% higher than the magnetic thermal group, which may be a result of the differences of magnetocaloric and photothermal
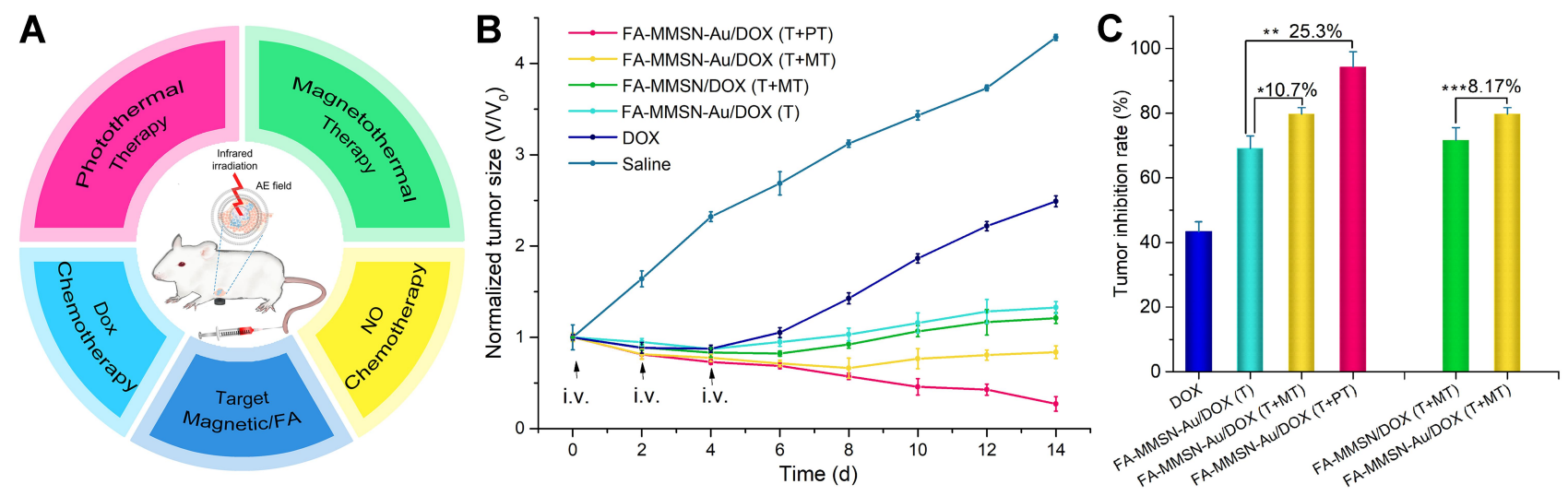

Figure 7 (A) Scheme of multi-therapy, (B) Tumor growth curves and (C) tumor inhibition curves of different treatments. Notes: $* P<0.05, * * P<0.05$, and $* * * P<0.05$. 
properties. As can be seen from the comparison of the FA-MMSN-Au/DOX (T+MT) and FA-MMSN/DOX ( T $+\mathrm{MT}$ ) group, Au's presence in the combination procedure of DOX chemotherapy and thermal therapy can contribute to tumor inhibition with an increment of $8.17 \%$.

\section{Distribution and Catalytic Ability of FA-MMSN-Au/DOX Carrier in vivo}

To verify that $\mathrm{Au}$ can act as a $\mathrm{NO}$ donor catalyst in vivo chemotherapy by releasing $\mathrm{NO}$ in a thermally controlled manner, the distribution of FA-MMSN-Au/DOX carrier and its catalytic ability in vivo was studied. As shown in Figure $8 \mathrm{~A}$, after the carrier was magnetically targeted at the tumor site for $30 \mathrm{~min}$ followed by hyperthermia procedure, the target site's temperature increased quickly. It reached more than $43^{\circ} \mathrm{C}$ in $300 \mathrm{~s}$ while the non-target part showed less change, suggesting the distribution of FA-MMSN-Au /DOX carrier was mainly at the target tumor site (Figure $8 \mathrm{~A})$. During the multiple therapy processes, the Au content in the tumor tissue is shown in Figure 8B. The Au content in the tumor tissue increased to $11.7 \mu \mathrm{g}$ after the third target and photothermal treatment. The release amount of NO catalyzed by the carrier in vivo increased with the number of treatments related to the accumulation of $\mathrm{Au}$ in tumor tissues. After three hyperthermia treatments, the amount of $54 \mu \mathrm{M}$ NO was checked out in urine in $96 \mathrm{~h}$ (Figure 8C). Without hyperthermia triggering $\mathrm{NO}$ release, the nitrogen content of mice's normal metabolism in the blank group was about two-thirds of the hyperthermia group. It can be seen that FA-MMSN-Au carrier exerted NO chemotherapy by releasing NO in a thermally controlled manner and hyperthermia effects in vivo through accumulation at targeting tumor site.

\section{Discussion}

\section{Effect of Au Catalysis on Tumor Proliferation}

In 6-14 days of the therapy, only FA-MMSN-Au/DOX (T $+\mathrm{PT})$ and $(\mathrm{T}+\mathrm{MT})$ groups combing chemo-thermotherapy with Au's presence showed a reduction in tumor volume, which was different from other groups. This reduction was related to tumor proliferation. Without inhibiting the tumor's proliferative ability, even if short-term combined therapy can cause tumor cell apoptosis within $48 \mathrm{~h}$ of the treatment, tumor cell expansion and even crazy growth of drug resistance would occur in later stages following tumor volume increase. Therefore, Au's presence has a suppressive effect on tumor proliferation in the combination of thermotherapy and DOX chemotherapy. As mentioned above, Au exerted NO chemotherapy and hyperthermia effects in vivo through accumulation and catalysis at targeting tumor sites; to be precise, it was NO that enhanced the inhibition of the proliferative process of combination DOX chemotherapy and hyperthermia. Without NO action, the FA-MMSN/DOX (T $+\mathrm{MT}$ ) group's tumor volume showed an obvious increase in 6-14 days. From the report that the hyperthermia combined NO chemotherapy could induce apoptosis and inhibit tumor proliferation, ${ }^{36}$ we can infer that Au catalyzing NO release contributes to reducing drug resistance and tumor proliferation, resulting in an increasing tumor inhibition rate of $8.17 \%$.

\section{Effect of Thermally Control Release NO on Tumor Apoptosis}

On the 6th day, the FA-MMSN-Au/DOX (T+MT) tumor growth curves showed a lower peak of tumor growth inhibition rate than that of the FA-MMSN-Au/DOX (T)
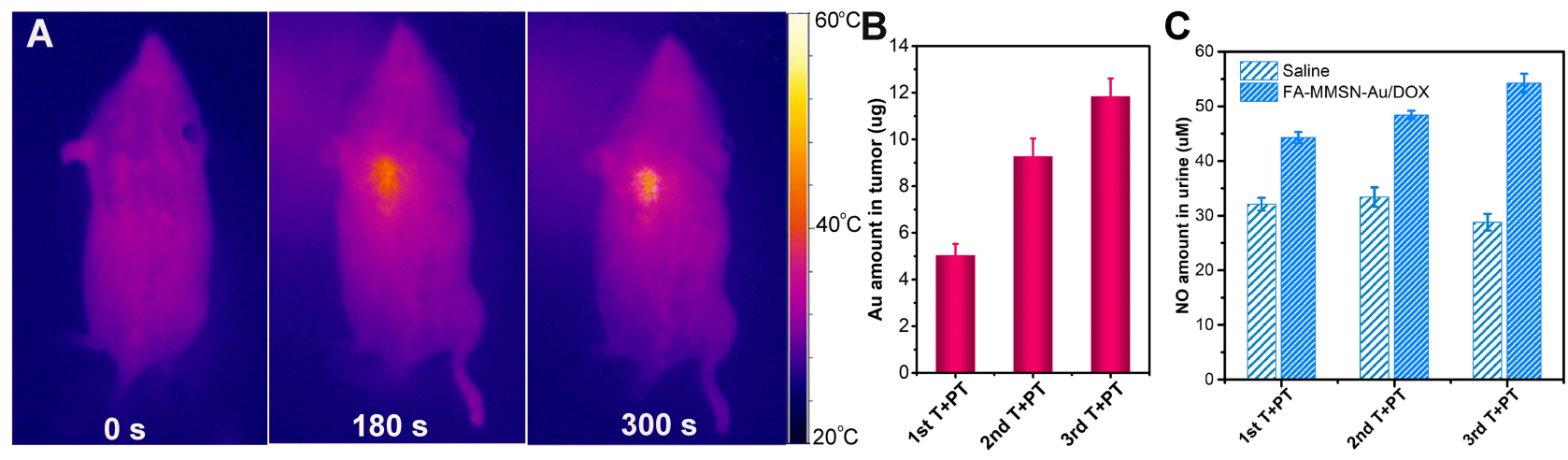

Figure 8 (A) Image of photothermal therapy; (B) ICP detection of gold ion concentration in tumor tissues; (C) NO amount in urine during 24 h after different times of target and photothermal treatments $(\mathrm{T}+\mathrm{PT})$. 
group, which could be ascribed to thermotherapy. For the T+MT group's heating process was stable, protein thermal tolerance may be generated, ${ }^{37}$ and less tumor damage was caused by heat. Therefore, the effect of NO thermally controlled release on tumor growth can be analyzed by comparison with FA-MMSN-Au/DOX (T) group and (T $+\mathrm{MT}$ ) group. Since the amount of gold-catalyzed NO release in vitro was temperature-dependent, the NO release amount at the hyperthermia temperature of $43^{\circ} \mathrm{C}$ was $1 / 3$ higher than that released at $37^{\circ} \mathrm{C}$. Therefore, it can be inferred that the amount of NO released by the Au catalysis in the T+MT group was greater than that of the $\mathrm{T}$ group. The group with a higher NO concentration has a higher apoptotic rate, which is consistent with previous studies. ${ }^{36}$ From the tumor growth curves comparison of the two groups, it can be seen that thermally control releasing NO causes a high apoptotic rate and results in tumor inhibition rate increasing by $10.7 \%$.

\section{Effect of Heating Rate on Tumor Survival Time}

On the 14th day, the FA-MMSN-Au/DOX $(\mathrm{T}+\mathrm{PT})$ tumor growth curves showed a lower peak of tumor growth inhibition rate than that of the FA-MMSN-Au/DOX $(\mathrm{T}+\mathrm{MT})$ group, which can be ascribed to the difference of magneto/ photothermal properties. For the MMSN-Au carrier, the heat production rate of photothermal performance was higher than that of magnetothermal performance under the experiment condition (Figure 5C). In vivo, it takes only $5 \mathrm{~min}$ for the tumor tissue to reach $43^{\circ} \mathrm{C}$ in photothermal therapy, and the time of heating the tumor site is $20 \mathrm{~min}$ for magnetic hyperthermia. In photothermal therapy, the tumor tissue temperature increases rapidly that may cause more damage to the tumor cells. However, the temperature rise of the tumor tissue under the magnetothermal condition is gentle and slow, leading to the tumor cells' heat tolerance. It has been reported that the formation of heat tolerance accompanied by overexpression of the BCL-2 gene reduces heat-induced apoptosis. ${ }^{38}$ We can infer that FA-MMSN-Au/DOX (T+PT) therapy with a higher heating rate character can increase tumor cell apoptosis than FA-MMSN-Au/DOX (T+MT) group with heat tolerance. Also, it should be noted that in the stage of 6-14 days, the tumor growth of the FA-MMSN /DOX $(\mathrm{T}+\mathrm{MT})$ group was stable. However, the tumor volume of the FA-MMSN/DOX $(\mathrm{T}+\mathrm{PT})$ group continued to decrease. This difference may be related to prolonged tumor survival. As mentioned above, heat tolerance may stimulate tumor growth, mainly prolonging cells' survival time. ${ }^{39} \mathrm{We}$ can infer that rapid-heat treatment is more helpful in increasing tumor inhibition rate by shortening survival time. Combined with rapid-heat treatment, the tumor inhibition rate of chemotherapy can be increased by $25.3 \%$.

\section{Conclusions}

We have constructed the FA-MMSN-Au/DOX platform as a NO donor that can thermally control released $\mathrm{NO}$ in vivo with a tumor inhibition rate of $94.3 \%$ in combining the targeted chemo-thermotherapy. This multifunctional platform catalyzed GSNO release NO in vitro and caused a high NO release concentration in vivo in thermotherapy, which increased the apoptotic rate and reduced tumor proliferation. The feature of extremely rapid hyperthermia due to $\mathrm{Fe}_{3} \mathrm{O}_{4}$ and $\mathrm{Au}$ twocomponent also caused tumor shortening survival time. The contribution of this paper is constructing an integrated therapeutic system as a NO donor that can thermally control released NO in vivo and verifying the effect of $\mathrm{Au}$ on tumor inhibition. As many critical biological functions of $\mathrm{NO}$ are related to its concentration, in the future, the real-time detection of different concentrations of $\mathrm{NO}$ in vivo and the damage to different tumor cells will be further studied.

\section{Funding}

This work is funded by the National Natural Science Foundation of China No. 81401501, Educational Department of Liaoning Province No. 2019LJC03 and No. LJQ2015110, Scholarship Council of China No. 20180820463, the College Student Funded and Innovation Program of Liaoning Province No. 2020101163039 and 201919163050, Young and Middle-aged Teachers Career Development Support Plan of Shenyang Pharmaceutical University No. ZQN2015013.

\section{Disclosure}

The authors report no conflicts of interest for this work.

\section{References}

1. Lee H, Han J, Shin H, Han H, Na K, Kim H. Combination of chemotherapy and photodynamic therapy for cancer treatment with sonoporation effects. J Control Release. 2018;283:190-199. doi:10. 1016/j.jconrel.2018.06.008

2. Zhang CT, Li J, Yang CX, et al. A pH-sensitive coordination polymer network-based nanoplatform for magnetic resonance imaging-guided cancer chemo-photothermal combination therapy. Nanomed Nanotech Biol Med. 2020;23:102071. doi:10.1016/j.nano.2019.102071 
3. Rui S, Chen M, Yang YY, et al. HAp@GO drug delivery vehicle with dual-stimuli-triggered drug release property and efficient combination therapy function against cancer. J Biomed Mater Res. 2019; 107A:2296-2309.

4. Zhao QF, Yang Y, Wang HL, Lei W, Liu YX, Wang SL. Gold nanoparticles modified hollow carbon system for dual-responsive release and chemo-photothermal combination therapy of tumor. $J$ Colloid Interface Sci. 2019;554:239-249. doi:10.1016/j.jcis.20 19.07.005

5. Zhang LW, Rong PF, Chen ML, Gao S, Zhu L. A novel single walled carbon nanotube (SWCNT) functionalization agent facilitating in vivo combined chemo/thermo therapy. Nanoscale. 2015;7(39):162 04-16213. doi:10.1039/C5NR03752B

6. Lei W, Sun CS, Jiang TY, et al. Polydopamine-coated mesoporous silica nanoparticles for multi-responsive drug delivery and combined chemo-photothermal therapy. Mat Sci Eng C. 2019;105:110103-110111. doi:10.1016/j.msec.2019.110103

7. Tonnessen-Murray CA, Frey WD, Rao SG, et al. Chemotherapyinduced senescent cancer cells engulf other cells to enhance their survival. J Cell Biol. 2019;218:3827-3844. doi:10.1083/jcb.20190 4051

8. Ganguly A, Chakraborty P, Banerjee K, Choudhuri SK. The role of a Schiff base scaffold, N-(2-hydroxy acetophenone) glycinate-in overcoming multidrug resistance in cancer. Eur J Pharm Sci. 2014;51:96-109. doi:10.1016/j.ejps.2013.09.003

9. Gholamreza K, Farhad JN, Abdolreza J, Keivan Z, Mohammad HF. Mechanisms of tumor cell resistance to the current targeted-therapy agents. Tumor Biol. 2016;37:10021-10039. doi:10.1007/s13277-0165059-1

10. Hoang TTT, Lee Y, Le TP, Park KD. Nitric oxide-releasing injectable hydrogels with high antibacterial activity through in situ formation of peroxynitrite. Acta Biomater. 2018;67:66-78. doi:10.1016/j.actbio. 2017.12.005

11. Albina JE, Reichner JS. Role of nitric oxide in mediation of macrophage cytotoxicity and apoptosis. Cancer Metastasis Rev. 1998;17:39-53. doi:10.1023/A:1005904704618

12. Fukumura D, Kshiwagi S, Jain RK. The role of nitric oxide in tumor progression. Nat Rev Cancer. 2006;6:521-534. doi:10.1038/ nrc1910

13. Lo HW, Hung MC. Nuclear EGFR signaling network in cancers: linking EGFR pathway to cell cycle progression, nitric oxide pathway and patient survival. Br J Cancer. 2006;94:184-188. doi:10.1038/sj. bjc. 6602941

14. Duan WW, Li J, Inks ES, et al. Design, synthesis, and antitumor evaluation of novel histone acetylase inhibitors equipped with a phenylsulfonylfuroxan module as a nitric oxide donor. $J$ Med Chem. 2015;58:4325-4338. doi:10.1021/acs.jmedchem.5b00317

15. Sudhesh P, Tamilarasan K, Arumugam P, Berchmans S. Nitric oxide releasing photo responsive nanohybrids as excellent therapeutic agent for cervical cancer cell lines. ACS Appl Mater Inter. 2013; 5:8263-8266. doi:10.1021/am402086m

16. Stevens E, Carpenter A, Shin J, Liu J, Der CJ, Schoenfisch MH. Nitric oxide-releasing silica nanoparticle inhibition of ovarian cancer cell growth. Mol Pharm. 2010;7:775-785. doi:10.1021/ mp9002865

17. Mimeault M, Jouy N, Depreux P, Hénichart JP. Combination antiproliferative and apoptotic effects induced by mixed epidermal growth factor receptor inhibitor ZD1839 and nitric oxide donor in human prostatic cancer cell lines. Prostate. 2005;62:187-199. doi:10.1002/pros.20138

18. Sarma M, Mondal B. Nitric oxide reactivity of copper (ii) complexes of bidentate amine ligands: effect of substitution on ligand nitrosation. Dalton T. 2012;41:2927-2934. doi:10.1039/c2dt11082b
19. Williams D, Lyn H. The mechanism of nitric oxide formation from S-nitrosothiols (thionitrites). Chem Commun. 1996;10:1085-1091. doi: $10.1039 / \operatorname{cc} 9960001085$

20. Zhou JL, Wang ZH, Li QP, et al. Hybridized doxorubicin-Au nanospheres exhibit enhanced near-infrared surface plasmon absorption for photothermal therapy applications. Nanoscale. 2015;7:586 9-5883. doi:10.1039/C4NR07279K

21. Li Q, Yuan Q, Zhao MH, et al. Au nanoclusters suppress chronic lymphocytic leukaemia cells by inhibiting thioredoxin reductase 1 to induce intracellular oxidative stress and apoptosis. Sci Bull. 2017;62:537-545. doi:10.1016/j.scib.2017.03.024

22. Xing YF, Kang TL, Luo XJ, Zhu JT, Wu P, Cai CX. Coral-shaped Au nanostructures for selective apoptosis induction during photothermal therapy. J Mat Chem B. 2019;7:6224-6231. doi:10.1039/C9TB01503E

23. Huang XH, Jain PK, EI-Sayed IH, EI-Sayed MA. Gold nanoparticles: interesting optical properties and recent applications in cancer diagnostics and therapy. Nanomedicine. 2007;2:681-693. doi:10.2217/ 17435889.2.5.681

24. Ma M, Chen HG, Chen Y, et al. Au capped magnetic core/mesoporous silica shell nanoparticles for combined photothermo-/chemotherapy and multimodal imaging. Biomaterials. 2012;33:989-998. doi:10.1016/j.biomaterials.2011.10.017

25. Wang YM, Ji X, Pang P, et al. Synthesis of Janus Au nanorods/polydivinylbenzene hybrid nanoparticles for chemo-photothermal therapy. J Mat Chem B. 2018;6:2481-2488. doi:10.1039/C8TB00233A

26. Chen CW, Syu WJ, Huang TC, et al. Encapsulation of $\mathrm{Au} / \mathrm{Fe} 3 \mathrm{O} 4$ nanoparticles into a polymer nanoarchitecture with combined near infrared-triggered chemo-photothermal therapy based on intracellular secondary protein understanding. J Mat Chem B. 2017;5:5774-5782. doi:10.1039/C7TB00944E

27. Jongnam P, Kwangjin A, Yosun H, et al. Ultra-large-scale syntheses of monodisperse nanocrystals. Nat Mater. 2004;3:891-895. doi:10.1038/nmat 1251

28. Che EX, Gao YK, Wan L, et al. Paclitaxel/gelatin coated magnetic mesoporous silica nanoparticles: preparation and antitumor efficacy in vivo. Micropor Mesopor Mater. 2015;204:226-234. doi:10.1016/j. micromeso.2014.11.013

29. He LP, Li WP, Qu XH, et al. The experimental study on biocompatibility of tendon suture. J Biomed Eng Res. 2004;4:236-239.

30. Zhang Y, Zhang Q, Che EX, et al. A novel magnetic mesoporous silicon composite combining the function of magnetic target drug delivery and magnetic-induction hyperthermia. Mater Technol. 2015;30(Supp18): B211-B215. doi:10.1179/17535557B15Y.000000010

31. Li J, Wang JH, Yang A, et al. Preparation and photothermal catalytic properties of gold nanospheres and nanorods. J Inorg Chem. 2018;34:1610-1614.

32. Robert JG, Jonathan DS, Benjamin JL. Photothermal effectiveness of magnetite nanoparticles: dependence upon particle size probed by experiment and simulation. Molecules. 2018;23:1234. doi:10.3390/ molecules 23051234

33. Asrin P, Roya S, Mehrdad M. Comparison study on the effect of gold nanoparticles shape in the forms of star, hallow, cage, rods, and Si-Au and Fe-Au core-shell on photothermal cancer treatment. Photodiagn Photodyn Ther. 2021;33:102144. doi:10.1016/j.pdpdt.2020.102144

34. Qin CC, Fei JB, Cai P, Zhao J, Li J. Biomimetic membrane-conjugated graphene nanoarchitecture for light-manipulating combined cancer treatment in vitro. $J$ Colloid Interface Sci. 2016;482:121-130. doi:10.1016/j.jcis.2016.07.031

35. Gomhor J, Alqaraghuli H, Kashanian S, Rafipour R, Mahdavian E, Mansouri K. Development and characterization of folic acid-functionalized apoferritin as a delivery vehicle for epirubicin against MCF-7 breast cancer cells. Artif Cell Nanomed B. 2018;46: S847-S854. doi:10.1080/21691401.2018.1516671 
36. Feng ZZ, Yang ZR, Li JC. Experiment research on the apoptosis of Hep-A cells induced by hyperthermia combined with ISDN. J Biomed Eng. 2004;37:391-397.

37. Wust P, Gneveckow U, Johannsen M, et al. Magnetic nanoparticles for interstitial thermotherapy-feasibility, tolerance and achieved temperatures. Int J Hyperthermia. 2006;22:673-685. doi:10.1080/ 02656730601106037
38. Salha Eldin AE, Inoue S, Tsukamoto S, Aoi H, Tsuda M. An association of Bcl-2 phosphorylation and Bax localization with their functions after hyperthermia and paclitaxel treatment. Int $J$ Cancer. 2003;103:53-60. doi:10.1002/ijc.10782

39. Vaux D, Cory S, Adams JM. Effects of Bcl-2 gene in the tumor culture in vitro. Nature. 1988;335:440-442. doi:10.1038/335440a0

\section{Publish your work in this journal}

The International Journal of Nanomedicine is an international, peerreviewed journal focusing on the application of nanotechnology in diagnostics, therapeutics, and drug delivery systems throughout the biomedical field. This journal is indexed on PubMed Central, MedLine, CAS, SciSearch ${ }^{\mathbb{B}}$, Current Contents ${ }^{\mathbb{B}} /$ Clinical Medicine, $^{2}$
Journal Citation Reports/Science Edition, EMBase, Scopus and the Elsevier Bibliographic databases. The manuscript management system is completely online and includes a very quick and fair peer-review system, which is all easy to use. Visit http://www.dovepress.com/ testimonials.php to read real quotes from published authors.

Submit your manuscript here: https://www.dovepress.com/international-journal-of-nanomedicine-journal 